\title{
A Case of Eosinophilic Granulomatosis with Polyangiitis
}

Anita Modi, MD and Lily Ackermann, MD

\section{INTRODUCTION}

Eosinophilic granulomatosis with polyangiitis (eGPA) is a small-and medium-sized-vessel vasculitis with multi-organ manifestations. Given the rarity of eGPA, patients are often misdiagnosed for decades and may initially present with life-threatening manifestations of late-stage disease. Therefore, it is important to raise awareness of this condition and its associated signs and symptoms. This case report serves to describe a classic presentation of a patient with eGPA, as well as to delineate the diagnostic workup, acute management, and early outpatient follow-up required.

\section{CASE PRESENTATION}

A 57-year-old male with a history of recently-diagnosed asthma (on chronic prednisone) presented to the emergency department at our hospital with a month-long history of shortness of breath and fatigue. He denied wheezing and chest tightness typical of his asthma flares, insisting that this isolated dyspnea was similar to the symptoms he experienced two months earlier when he presented to an outside hospital and was diagnosed with symptomatic anemia.

He reported that he was in perfect health until one year prior to presentation when he developed progressively worsening respiratory symptoms. After evaluation by a pulmonologist, he was started on budesonide-formoterol twice daily in conjunction with his rescue inhaler. However, he had persistent symptoms over the subsequent six months, so was ultimately started on prednisone $30 \mathrm{mg}$ $\mathrm{PO}$ daily. At the time, he was noted to have an elevated IgE and eosinophil count of $104 \mathrm{IU} / \mathrm{mL}$ and 900 cells/uL, respectively. Though he improved dramatically with the addition of systemic steroids, he began to develop generalized fatigue, weakness, chills, night sweats, and migratory polyarthralgias a couple months later. His primary care physician suspected Lyme disease and started doxycycline without symptomatic improvement. He began taking ibuprofen several times a day for the diffuse joint pains. As the patient's shortness of breath and fatigue worsened, a complete blood count was obtained, and his hemoglobin was found to be $6 \mathrm{~g} / \mathrm{dL}$. He was admitted to an outside hospital where he received a blood transfusion and underwent an upper endoscopy and colonoscopy which did not demonstrate active bleeding. He was discharged with instructions to avoid further NSAID use.

In the emergency department at our hospital, his hemoglobin was $7.4 \mathrm{~g} / \mathrm{dL}$ so he received a blood transfusion for symptomatic anemia, which was suspected to be due to an upper gastrointestinal bleed caused by NSAID- and steroid-induced peptic ulcer disease. His chest x-ray demonstrated patchy bilateral airspace opacities throughout both lung fields concerning for multifocal pneumonia. He was also started on ceftriaxone in conjunction with his ongoing doxycycline therapy for treatment of superimposed community-acquired pneumonia. One hour after receiving one unit of blood, the patient developed increased work of breathing with hypoxia that did not improve with diuresis. A computed tomography (CT) scan of the chest without contrast was ordered to delineate possible hemorrhage or atypical infection contributing to his deteriorating presentation. It demonstrated multifocal airspace opacities in a peri-bronchial vascular distribution with relative sparing of the left lower lobe and subpleural spaces, findings consistent with diffuse alveolar hemorrhage (DAH)" and noncardiogenic pulmonary edema (Figure 1).
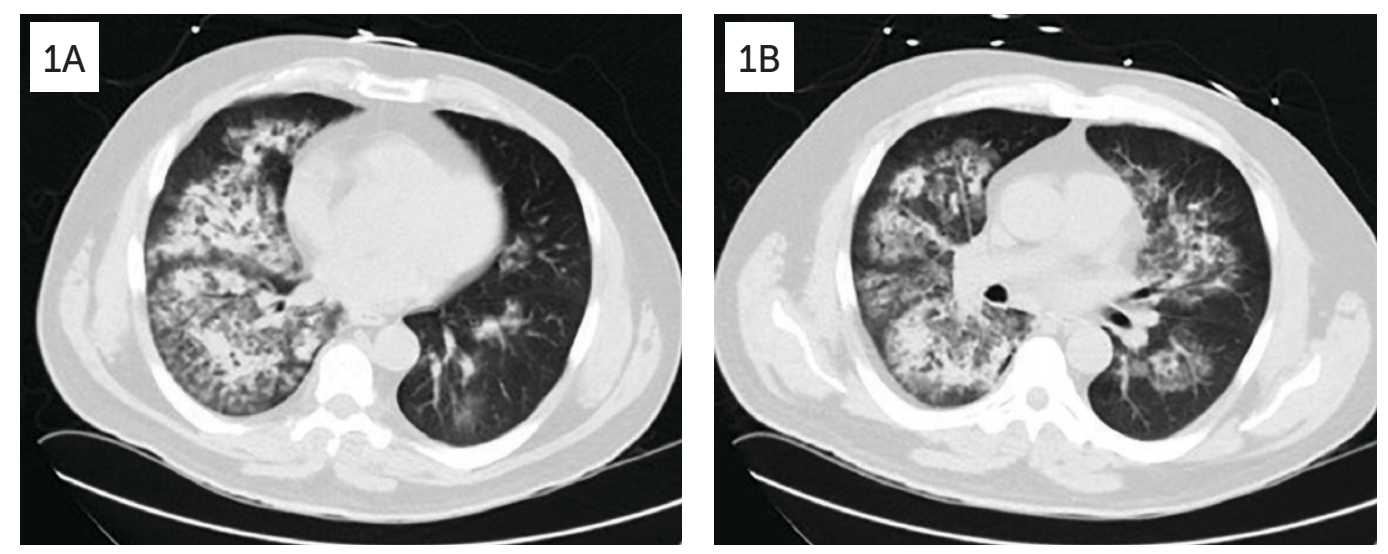

Figure 1. Images from the patient's CT scan of the chest demonstrates multifocal airspace opacities in a peri-bronchial vascular distribution with relative sparing of the left lower lobe and subpleural spaces. 
The patient's systemic symptoms and imaging was suspicious for an underlying vasculitis complicated by diffuse alveolar hemorrhage and gastroenteritis. He underwent bronchoscopy which demonstrated diffuse bloody secretions in the right upper lobe and lateral basilar segment of the right lower lobe. Cytology demonstrated marked acute inflammation with no evidence of infection. Differential cell count did not demonstrate eosinophils as would be expected in eGPA, but this may be related to the patient's chronic steroid use pre-procedure. Laboratory studies were significant for positive C-ANCA and PR-3 antibody with a titer of 4.3. In conjunction with the patient's elevated IgE and eosinophilia from six months earlier, this lab work helped to confirm the patient's suspected diagnosis of eGPA.

On further discussion, the patient acknowledged that he had experienced recurrent sinusitis and exerciseinduced asthma for one decade prior to initial presentation. His sporadic episodes were managed by his primary care physician until one year prior to admission when he sought evaluation by a pulmonologist and was ultimately started on chronic prednisone, which alleviated his respiratory symptoms and likely palliated his underlying vasculitis.

\section{OUTCOME}

The patient was pulsed with intravenous methylprednisolone 1 gram daily for 3 days, followed by oral prednisone $1 \mathrm{mg} / \mathrm{kg}$ daily. He also received his first dose of rituximab with the remaining three weekly infusions to be scheduled as an outpatient, and two sessions of inpatient plasmapheresis given his diffuse alveolar hemorrhage. These interventions resulted in prompt alleviation of his respiratory symptoms and migratory polyarthralgias, and he was weaned to room air prior to discharge. Six months after discharge, his prednisone has been tapered to $40 \mathrm{mg}$ daily, his repeat CT scan of the chest demonstrates resolution of focal airspace opacities initially observed in the setting of $\mathrm{DAH}$, and his PR-3 antibody titer is negative.

\section{DISCUSSION}

eGPA is characterized by the histological findings of necrotizing vasculitis as well as eosinophilic infiltrates and granulomas in tissues. ${ }^{1}$ The American College of Rheumatology has since defined six diagnostic criteria, of which four should be present in a patient with suspected eGPA: asthma, greater than 10\% peripheral eosinophilia, neuropathy, non-fixed pulmonary infiltrates, paranasal sinus abnormalities, and extravascular eosinophils. Our patient had a long history of recurrent sinusitis and asthma prior to presentation, eosinophilia, and pulmonary infiltrates. The disease progresses over the course of decades, classified into three distinct phases: the prodromal phase that is associated with allergic rhinitis and asthma; eosinophilic infiltration of multiple organs including the lungs and gastrointestinal tract; and granulomatosis resulting in life-threatening disease. ${ }^{2}$

Clinical presentations of eGPA differ based on associated ANCA positivity. ANCA-negative patients typically present with more frequent cardiomyopathy, while ANCA-positive patients present with mononeuritis multiplex and glomerulonephritis. Poorer prognoses can be anticipated for patients with documented gastrointestinal, renal, or cardiac manifestations of the disease, meriting screening endoscopies, urinalyses, and transthoracic echocardiograms of in-patients with newly-diagnosed eGPA. ${ }^{3}$ Commonly noted laboratory abnormalities include serum eosinophilia, elevated IgE levels, and P-ANCA of anti-MPO specificity. However, up to $40 \%$ of patients with eGPA demonstrate positive C-ANCA of anti-PR-3 specificity. Extravascular eosinophilia (e.g. in bronchoalveolar lavage fluid) and biopsy demonstrating granulomas aid in confirming the diagnosis of eGPA. Despite these objective features, eGPA is essentially a clinical diagnosis based on symptom presentation and the presence of eosinophilia.

Recommendations on treatment of eGPA depend on the severity of disease and organ involvement. Glucocorticoids are first-line treatment for EGPA and those with severe documented gastrointestinal, renal, pulmonary, or cardiac involvement should be prescribed an additional immunosuppressant (e.g. rituximab, cyclophosphamide). Though plasma exchange is not merited for every patient with newly-diagnosed eGPA, it may successfully forestall progressive glomerulonephritis in ANCA-positive patients with severe diffuse alveolar hemorrhage and renal insufficiency.

\section{KEY POINTS}

Given the life-threatening complications of this rare condition, it is important to raise awareness about eGPA. This is a classic presentation of eGPA in a patient with a decades-long asthma history, eosinophilia, new lung and gastrointestinal manifestations, and systemic symptoms. His serologies were significant for positive C-ANCA of anti-PR-3 specificity. His clinical course included screening for gastrointestinal, renal, and cardiac involvement as well as initiating steroid therapy, plasmapheresis, and rituximab.

\section{REFERENCES}

1. MouthonL, Dunogue B, Guillevin L. Diagnosis and Classification of Eosinophilic Granulomatosis with Polyangiitis (formerly named Churg-Strauss Syndrome). Journal of Autoimmunity. 2014:55(94): 99-103.

2. Lanham JG, Elkon KB, Pusey CD, Hughes GR. Systemic Vasculitis with Asthma and Eosinophilia: A Clinical Approach to the Churg-Strauss Syndrome. Medicine. 1984;63(2): 65-81.

3. Groh M, Pagnoux C, Baldini C, et al. Eosinophilic Granulomatosis with Polyangiitis (Churg-Strauss) (EGPA) Consensus Task Force Recommendations for Evaluation and Management. European Journal of Internal Medicine. 2015;26(7): 545-553. 